\title{
Comparison of the Joel-Cohen-based technique and the transverse Pfannenstiel for caesarean section for safety and effectiveness: A systematic review and meta-analysis
}

\author{
Alireza Olyaeemanesh ${ }^{1}$, Elahe Bavandpour ${ }^{2 *}$, Mohammadreza Mobinizadeh ${ }^{1}$, Mansoor Ashrafinia ${ }^{3}$, \\ Maryam Bavandpour ${ }^{2}$, Mojtaba Nouhi ${ }^{4}$
}

Received: 21 Jan 2017

Published: 4 Sep 2017

\section{Abstract}

Background: Caesarean section (C-section) is the most common surgery among women worldwide, and the global rate of this surgical procedure has been continuously rising. Hence, it is significantly crucial to develop and apply highly effective and safe caesarean section techniques. In this review study, we aimed at assessing the safety and effectiveness of the Joel-Cohen-based technique and comparing the results with the transverse Pfannenstiel incision for C-section.

Methods: In this study, various reliable databases such as the PubMed Central, COCHRANE, DARE, and Ovid MEDLINE were targeted. Reviews, systematic reviews, and randomized clinical trial studies comparing the Joel-Cohen-based technique and the transverse Pfannenstiel incision were selected based on the inclusion criteria. Selected studies were checked by 2 independent reviewers based on the inclusion criteria, and the quality of these studies was assessed. Then, their data were extracted and analyzed.

Results: Five randomized clinical trial studies met the inclusion criteria. According to the exiting evidence, statistical results of the Joel-Cohen-based technique showed that this technique is more effective compared to the transverse Pfannenstiel incision. Metaanalysis results of the 3 outcomes were as follow: operation time (5 trials, 764 women; WMD -9.78; 95\% CI:-14.49-5.07 minutes, $\mathrm{p}<0.001$ ), blood loss ( 3 trials, 309 women; WMD -53.23 ml; 95\% -CI: 90.20-16.26 ml, p= 0.004), and post-operative hospital stay (3 trials, 453 women; WMD -.69 day; 95\% CI: 1.4-0.03 day, $\mathrm{p}<0.001)$. Statistical results revealed a significant difference between the 2 techniques.

Conclusion: According to the literature, despite having a number of side effects, the Joel-Cohen-based technique is generally more effective than the Pfannenstiel incision technique. In addition, it was recommended that the Joel-Cohen-based technique be used as a replacement for the Pfannenstiel incision technique according to the surgeons' preferences and the patients' conditions.

Keywords: Caesarean Section (C-section), Joel-Cohen, Pfannenstiel, Misgav-Ladach, Modified Misgav-Ladach

Copyright@ Iran University of Medical Sciences

Cite this article as: Olyaeemanesh A, Bavandpour E, Mobinizadeh M, Ashrafinia M, Bavandpour M, Nouhi M. Comparison of the Joel-Cohen-based technique and the transverse Pfannenstiel for caesarean section for safety and effectiveness: A systematic review and meta-analysis. Med $J$ Islam Repub Iran. 2017 (4 Sep);31:54. https://doi.org/10.14196/mjiri.31.54

\section{Introduction}

Caesarean section (C-section) is the action of taking out the fetus, placenta, and membranes through making incisions on the abdomen and uterus walls (1). This surgical procedure is one of the most common abdominal surgeries in both developed and developing countries (2). More to the point, childbirth is one of the most delicate and prominent services delivered in the health care systems in all societies, and because any kind of service should be

Corresponding author: Elahe Bavandpour, el.bavandpour@gmail.com

1. National Institute for Health Research, Tehran University of Medical Sciences, Tehran, Iran.

2. Tehran University of Medical Sciences, Tehran, Iran.

3. Arash University Hospital, Tehran University of Medical Sciences, Tehran, Iran

4. School of Health Management and Information Sciences, Iran University of Medical Sciences, Tehran, Iran. properly provided with the least cost and physical-mental conditions, Caesarean section, as one of the modes of childbirth, is no exception to this rule. To bring about the best results for the mother and her newborn, it has been estimated that the maximum acceptable level of performing a $\mathrm{C}$-section is $5 \%$ to $15 \%$ (3). C-section increases the mortality and morbidity for the mother and her newborn and also increases the cost of childbirth. Therefore, it is

$\uparrow$ What is "already known" in this topic:

Systematic review and meta-analysis of these two methods of cesarean section were conducted by researchers. These studies results showed that Joel-Cohen based' methods have advantages compared to Pfannenstiel.

$\rightarrow$ What this article adds:

This study updates the previous systematic review and metaanalysis on this topic. Results too show that Joel-Cohen based' methods have advantages compared to Pfannenstiel. 
crucial to reduce the rate of $\mathrm{C}$-section or use safer techniques (4).

A wide range of various methods for conducting cesarean is reported in the literature. Some researches targeting this subject provide little details on the operation, while others present major breakthroughs (5). Different techniques are used to perform a $\mathrm{C}$-section, and some of them have been evaluated through randomized clinical trials. Abdominal surgical incisions used in C-section fall into 2 categories:

(1) Vertical Incision (Midline, Paramedian);

(2) Transverse Incision (Pfannenstiel, Pelosi Maylard, Cherney and Joel-Cohen).

Each of these techniques has specific advantages, while suffering from different disadvantages. Therefore, choosing an incision depends on many factors such as the patient's health condition and the surgeon's choices (6).

This study aimed at establishing an evidence-based argument about safety and effectiveness of the Joel-Cohenbased technique as a new and presumably safe technique for C-section compared to the transverse Pfannenstiel incision.

Moreover, this study followed a comprehensive approach to conduct an up-to-date systematic review and meta-analytic comparison on all available studies.

\section{Methods}

Type of Study: All clinical trials, reviews, systematic reviews, and meta-analysis based on PICO in both Persian and English languages were reviewed in this study. Then, the required data (effectiveness, safety, etc.) were extracted.

\section{PICO}

Participants: Pregnant women due for delivery by Csection

Interventions: Joel-Cohen based caesarean section (CS)

Comparative: Pfannenstiel caesarean section (CS)

Outcome: operating time, blood loss volume, and the postoperative hospital stay for the mother

\section{Search Strategy}

To meet the aforementioned goals of this work, one of the most important steps was to conduct a comprehensive search for evidence in other countries. To do a comprehensive search in the target databases, it was necessary to find the right keywords including the PICO of the study. Thus, the following keywords were determined:

Caesarean Section (C-section); Joel-Cohen-based; Pfannenstiel Incision; Misgav-Ladach; and Modified MisgavLadach.

In addition, the E-library of Tehran Medical School and other databases such as PubMed, Cochran, DARE, and Ovid MEDLINE were searched. Table 1 shows details of search strategy for PubMed, Cochran and Ovid Medline databases.

Furthermore, to increase the precision of the search and find the largest number of articles, the search engines of Google, Google Scholar, and other Persian databases such as SID, Irandoc, Iranmedex, and mag Iran were used

\begin{tabular}{|c|c|}
\hline No. & PUB MED \& Cochran Search Strategy \\
\hline$\# 1)$ & "Pfannenstiel" \\
\hline$\# 2)$ & Mesh descriptor "Pfannenstiel" explode all trees \\
\hline \#3) & $(\# 1$ or \#2) \\
\hline \#4) & "Joel-Cohen" \\
\hline$\# 5)$ & $\begin{array}{l}\text { "Mesh descriptor Joel-Cohen explode all trees System- } \\
\text { atic Review" }\end{array}$ \\
\hline$\# 6)$ & $(\# 4$ or $\# 5)$ \\
\hline$\# 7)$ & $(\# 3$ and \#6) \\
\hline$\# 8)$ & "Caesarean section" \\
\hline \#9) & Mesh descriptor "Caesarean section" explode all trees \\
\hline \#10) & (\#8 and \#9) \\
\hline \#11) & $(\# 7$ and \#10) \\
\hline No. & Ovid Medline Search strategy \\
\hline$\# 1)$ & “c/s”.mp \\
\hline$\# 2)$ & "Joel-Cohen".mp \\
\hline$\# 3)$ & "misgava".mp \\
\hline \#4) & $(\# 2$ or $\# 3)$ \\
\hline \#5) & (\#1 and \#4) \\
\hline \#6) & "Pfannenstiel".mp \\
\hline$\# 7)$ & $(\# 1$ and $\# 6)$ \\
\hline \#8) & (\#5 and \#7) \\
\hline \#9) & "S.R".mp \\
\hline \#10) & rev \$.mp \\
\hline$\# 11)$ & $(\# 8$ and $\# 5)$ \\
\hline
\end{tabular}

(until March 2015).

\section{Definitions}

Pfannenstiel Incision Technique: The Pfannenstiel incision technique was introduced by Pfannenstiel in 1900.This technique is a curvilinear incision $(10-15 \mathrm{~cm})$ just above the pubic symphysis in which the rectus and fascia sheaths are incised separately (5).

Joel-Cohen-based Technique: The Joel-Cohen-based technique was introduced for performing abdominal surgery by Professor Joel Cohen in 1974.This technique has been widely used for $\mathrm{C}$-section ever since, but has gone through many modifications over time. The modified form of this technique, known as Misgav Ladach, was introduced by Professor Michael Stark in 1998 (7).

This technique is a straight transverse incision located 3 centimeters above the symphysis pubic and below the anterior superior iliac spines (above the Pfannenstiel incision). The tissues and fascia are spread apart about 2 to 3 centimeters in the midline, where it is free of large blood vessels and the incision is more broadened with 2 fingers (a blunt dissection). Then, the vertical rectus muscles are separated, the peritoneum is opened transversely with fingers. After the baby and placenta are extracted, the womb is sewn by single-layer suturing. However, the parietal and visceral layers of the peritoneum are not sutured (7).

\section{Inclusion and Exclusion Criteria}

Reviews, systematic reviews, and randomized clinical trial studies comparing the Joel-Cohen-based technique and the transverse Pfannenstiel incision were selected based on the inclusion criteria.

\section{Assessment of Methodological Quality of the Included} Studies

Several articles were selected and evaluated by 2 experts based on their relevance to the topic of this work for 


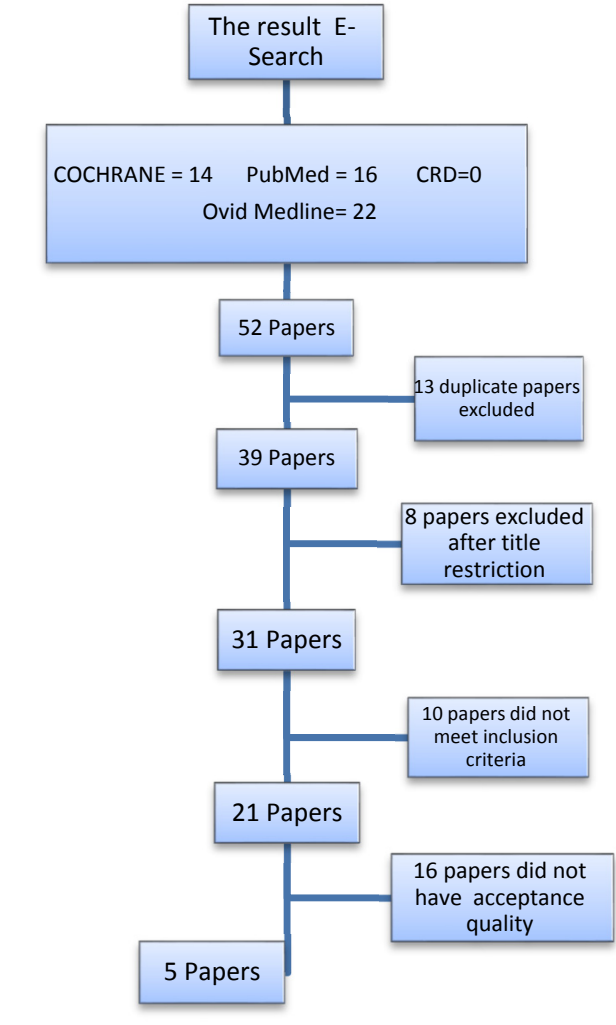

Fig. 1. Flow of the Papers through the Study

methodology and quality of study. The Critical Appraisal Skills Program (CASP) checklist was used to evaluate the RCT studies (Fig.1).

\section{Data Extraction and Analysis}

Results of CASP checklist proved that 5 RCT studies had sufficient quality. Therefore, the data of these studies were extracted. Then, the Rev-Man Version 5 software was used to analyze the data.

\section{Results}

According to the criteria of the meta-analysis study (PI$\mathrm{CO}), 5$ clinical trials (7-11) were selected to find evidence of the effectiveness of the Joel-Cohen-based technique compared to the traditional techniques.
In the selected studies, the interventions were 3 incision techniques: Joel-Cohen, Misgav-Ladach, and Modified Misgav-Ladach. These techniques have been named after the surgeons who introduced them for the first time.

All these new modified techniques fall into the category entitled 'Joel-Cohen-based technique' with 3 subcategories:

Joel-Cohen: Entering the abdomen and uterine with Joel-Cohen incision, closing the uterine with interrupted stitches while peritoneum is not closed, and suturing the skin with subcutaneous stitches (6).

Misgav-Ladach: Entering the abdomen and uterine with Joel-Cohen-based incision, single-layer closure of the uterine with continuous stitches excluding the peritoneum, and suturing the skin with interrupted stitches (6).

Modified Misgav-Ladach: All the above-mentioned steps are included in this technique, but the skin is tied up with subcutaneous stitches using different methods (6). In addition, the uterine (single layer) is sutured with noncontinuous stitches, the visceral layer of the peritoneum is tied up, and the womb is closed in 2 layers (non-locked) (5), or the skin is opened at the Pfannenstiel incision level for cosmetic reasons (6).

In this study, selected articles were categorized in the above-mentioned subgroups according to the type of intervention, and no significant difference was found between the subgroups after performing the meta-analysis. The Meta-analysis results were verified by the review conducted by Mathai et al. and Hofmeyr et al.

Based on evidence, the Joel-Cohen-based technique brings many advantages for users and service providers, some of which are as follow:

1. Less blood loss

2. Shorter surgical time

3. Sooner return to action

4. Less need for antipyretic medication

5. Longer time before taking the first dose of analgesia

6 . Earlier oral feeding

7. Less postoperative pain

8. Faster wound healing

9. Five-to-seven-minute Apgar score

10. Return of the intestine performance

11. Earlier discharge from hospital, etc. (7).

The outcomes of the studies on this subject are vast and different. Hence, 3 common outcomes of operating time,

Table 2. The Details of the Clinical Trials Eligible for the Meta-analysis on Efficiency, Effectiveness, and Safety of the Joel-Cohen-based Technique Versus the Transverse Pfannenstiel Incision for Caesarean Section

\begin{tabular}{|c|c|c|c|c|c|c|}
\hline Row & Author/Year & $\begin{array}{l}\text { The Type } \\
\text { of Study }\end{array}$ & Sample Population & Intervention Method & $\begin{array}{l}\text { Comparison } \\
\text { Method }\end{array}$ & The Intended Consequences \\
\hline 1 & Ddrj (1999) & RCT & 50 & Misgav-Ladach & Pfannenstiel & $\begin{array}{l}\text { Operating time, the contracted } \\
\text { blood volume and the number of } \\
\text { the hospitalization days }\end{array}$ \\
\hline 2 & Ferrari (2001) & RCT & 158 & Misgav-Ladach & Pfannenstiel & $\begin{array}{l}\text { Operating time and the contracted } \\
\text { blood volume }\end{array}$ \\
\hline 3 & Mathai (2002) & RCT & 101 & Joel-Cohen incision & Pfannenstiel & $\begin{array}{l}\text { Operating time, the contracted } \\
\text { blood volume and the number of } \\
\text { the hospitalization days }\end{array}$ \\
\hline 4 & Saha (2012) & RCT & 302 & Misgav-Ladach & Pfannenstiel & $\begin{array}{l}\text { Operating time and the number of } \\
\text { the hospitalization days }\end{array}$ \\
\hline 5 & Washam (2013) & RCT & 153 & Joel-Cohen incision & Pfannenstiel & Operating time \\
\hline
\end{tabular}


blood loss volume, and the number of postoperation hospital stay days were selected to facilitate the investigation, while delivering an effective evaluation. These outcomes are analyzed through the Rev- Man Version 5 software. The resultant observations are as follow:

(1) According to the Forest plot (Fig. 2), the operating time is measured in each of the 5 selected studies. Based on the findings, 764 women eligible for Caesarean section were studied (386 women were in the intervention group and 378 in the control group), and due to the heterogeneity of the results of studies, the random-effects model was used. For this outcome, $I^{2}=99 \%$ was calculated. Therefore, studies' results had high heterogeneity, and the duration of surgery in the intervention technique was shorter than that of the Pfannenstiel technique (weighted mean difference (WMD) is $-9.78 ; 95 \%$ CI: -14.49 to -5.07 minutes; $p<0.001$ ), which means that the mother and the newborn were less exposed to the side effects of unconsciousness, and the duration of surgery was shorter by $20 \%$. This result is consistent with that of the systematic review conducted by Mathai et al. and Hofmeyr et al. (12,
13). However, the results of these systematic review studies revealed a larger value. Egger's regression was used to check the possibility of publication bias. Results of Egger's test proved that the possibility of publication bias was not significant $(p=0.28)$.

(2) According to the Forest plot (Fig. 3) the lost blood volume was measured in each of the 3 selected studies. Based on the findings, 309 women eligible for Caesarean section were studied (159 women were in the intervention group and 150 in the control group), and because of the heterogeneity of the variance, the fixed effects model was used in the random effects model. The results demonstrated that the levels for this outcome $I^{2}=47 \%$, was calculated. Therefore, the studies' results represented moderate heterogeneity $(p=0.005)$. Lost blood volume in the intervention technique was less than that of Pfannenstiel technique (WMD $-53.23 \mathrm{~mL}$; 95\% CI: 90.20-16.26 $\mathrm{mL}, \mathrm{p}=$ $0.004)$. This result is consistent with the results of the systematic review conducted by Mathai et al. and Hofmeyr et al. $(12,13)$. To check the possibility of bias, the result of these systematic review studies showed a larger value.

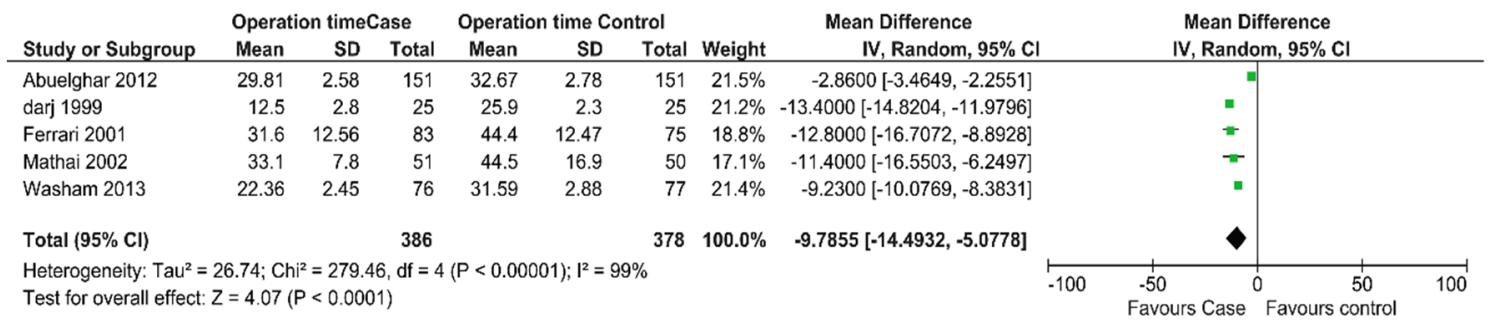

Fig. 2. The Forest Plot of the Efficiency and Effectiveness of the First Consequence (Operating Time)

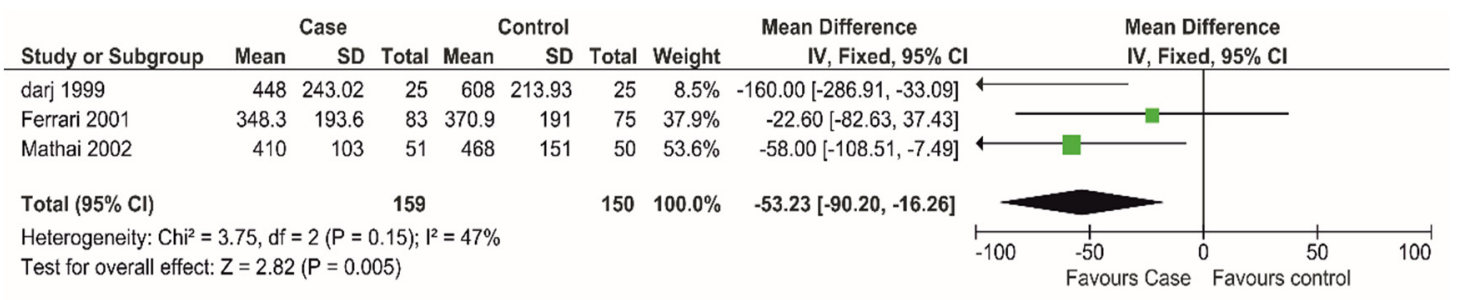

Fig. 3. The Forest Plot of the Efficiency and Effectiveness of the Second Consequence (the Contracted Blood Volume)

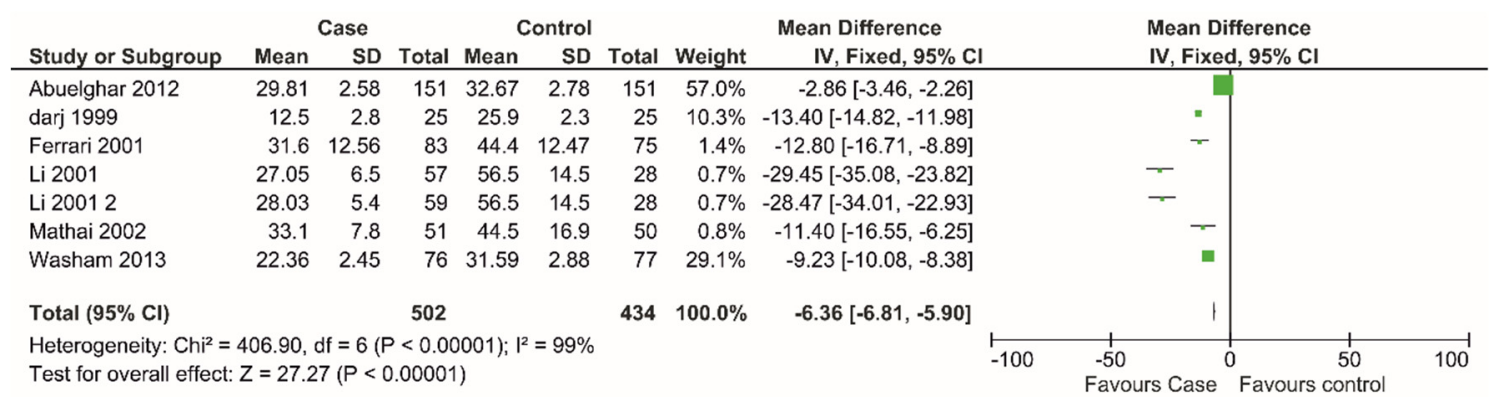

Fig. 4. The Forest Plot of the Efficiency and Effectiveness of the Third Consequence (the Number of Hospitalization Days) 
Egger's test was used to check the possibility of publication bias. Results of Egger's test revealed that the possibility of publication bias was not significant $(p=0.46)$.

However, given that the difference between the lost blood volumes in all studies was about $100 \mathrm{cc}$, which was not significant for mothers without anemia, although it was highly prominent for mothers suffering from anemia.

(3) According to the Forest plot (Fig. 4), the number of hospitalization days has been studied in 3 of the selected reviews. Based on the findings, 453 women eligible for Caesarean sections were studied (227 women were in the intervention group and 226 in the control group), and because of the heterogeneity of the variance, the random effects model was used. For this outcome $I^{2}=99 \%$ was calculated. Therefore, studies' results had high heterogeneity $(p<0.001)$. The number of hospitalization days in the intervention technique was less than that of Pfannenstiel technique (3 trials, 453 women; weighted mean difference (WMD) -.69 day; 95\% CI: with a confidence interval-1.4 to 0.03 day, $\mathrm{p}=0.00001$ ). This result is consistent with the results of the systematic review conducted by Mathai et al. and Hofmeyr et al. $(12,13)$.

However, the result of these systematic review studies revealed a larger value. Egger's regression were used to test publication bias $(p=0.25)$. The results of Egger's test showed that publication bias was not significant.

\section{Discussion}

The results of conducting systematic review and Metaanalysis on the results of the clinical trials verified the efficiency of the intervention methods in reducing the level of cesarean section side effects. In a review and meta-analysis conducted in the Cochrane scientific institution in 2009, more than 24 consequences were evaluated and further studies were used for the consequences. The present study suggests that the possibility of bias in the selected studies is minimal, and this issue encourages us to prefer the intervention method over the routine method.

In addition to the studies that entered this systematic review, numerous studies and documentation, eg, RHL WHO, were included in this report. "The evidence from this review suggests a beneficial effect of the Joel-Cohenbased techniques (14).

The Joel-Cohen method has more advantages than the traditional Pfannenstiel incision technique in short-term consequences, eg, less fever, shorter surgical time, less blood loss, less pain, longer time to take the first dose of painkiller, less infection, faster return to action, etc. (13). In addition to the studies that have examined the shortterm side effects of methods, some others have checked the long-term side effects of methods as well.

In an Iranian study conducted by Ghahiry et al. (19992008), 112 women undergone caesarean section were placed in 2 groups: one group had the Cesarean section with the Joel-Cohen technique and the other with Pfannenstiel incision technique. Moreover, this 10-year study was conducted to check and compare the long-term side effects of the 2 above-mentioned techniques such as pelvic adhesion, open incision mark (hernia), and chronic pelvic pain. In addition, the results of the same study demonstrated that the levels of adhesion in the filmy and dense type Pfannenstiel incisions were $50 \%$ and $17 \%$, respectively. Also, the levels of adhesion in the filmy and dense type Joel-Cohen technique were $50 \%$ and $12 \%$, respectively. Furthermore, the pelvis and hernia pains were significantly less compared to the Pfannenstiel technique $(17.2 \%$ versus $35 \%)$ (15).

On the other hand, in a prospective study conducted by Dumas et al. (2009) on 5123 women undergone caesarean section (43.2\% with the Joel-Cohen technique and 56.8\% with Pfannenstiel incision technique), the results revealed that the rates of hospital-acquired infections and endometriosis were more frequently observed in the Joel-Cohen technique compared to the Pfannenstiel incision technique (4.5\% versus $3.3 \%)$.

Thus, it was concluded that the new incision technique was a risk factor for endometriosis (16).

\section{Conclusion}

In spite of side effects of Joel-Cohen technique, it has been proven to be more effective compared to the Pfannenstiel incision method. Therefore, it is suggested this technique be used based on the surgeon's preferences and the patient's conditions as an alternative with the aim of meeting the interests of the patient and saving resources.

\section{Conflict of Interests}

The authors declare that they have no competing interests.

\section{References}

1. Zamani-Alavijeh F, Shahry P, Kalhory M, Haghighizadeh M H, Sharifirad G R, Khorsandi M. Identification of factors related to elective cesarean labor: a theory-based study. Daneshvar Med. 2012; 19 (96) :110.

2. Changizy N, Farahani Z. The Caesarean section in the world, the expansion in the extent and causes: Review.NAIGO2013; 1(8) :9-14

3. Amir Farahani L, Shavazi Abbasi MJ. Study of changes in the rate of cesarean section in Iran and demographic factors associated with it in the past three decades. J Med Fasa. 2012;2(3):127-134.

4. Dawary M, Marathi MR, Qureshi Z, Mokhtari M. The relationship between economic, social and prevalence of cesarean delivery in nulliparous women who referred to the training center Nick asked Rafsanjan. Health Info Manag .2011; 7(8): :958-965

5. Stark M, Chavkin Y, Kupfersztain C, Guedj P, Finkel AR. Evaluation of combinations of procedures in cesarean section. Int J Gyn \& Obst $.1995 ; 48(3): 273-6$.

6. Hofmeyr GJ, Mathai M, Shah AN, Novikova N. Techniques for caesarean section. Cochr Lib. $2008 \mathrm{Apr}$

7. Ferrari AG, Frigerio LG, Candotti G, Buscaglia M, Petrone M, Taglioretti A, Calori G. Can Joel-Cohen incision and single layer reconstruction reduce cesarean section morbidity? International journal of gynecology \& obstetrics. 2001 Feb 1;72(2):135-43

8. Darj E, Nordstrom ML. The Misgav Ladach method for cesarean section compared to the Pfannenstiel method. Acta obstetricia et gynecologica Scandinavica. 1999 Jan 1;78(1):37-41.

9. Mathai M, Ambersheth S, George A. Comparison of two transverse abdominal incisions for cesarean delivery. Int $\mathrm{J}$ Gyn \& Obst. 2002;78:47-9.

10. Saha SP, Bhattarcharjee N, Mahanta SD, Naskar A, Bhattacharyya SK. A randomized comparative study on modified Joel-Cohen incision versus Pfannenstiel incision for cesarean section. J Turk Ger Gyn Associa 2013;14(1):28.

11. Abuelghar WM, El-bishry G, Emam LH. Caesarean deliveries by Pfannenstiel versus Joel-Cohen incision: A randomised controlled trial. J Turk Ger Gyn Associa. 2013;14(4):194.

12. Mathai M, Hofmeyr GJ, Mathai NE. Abdominal surgical incisions for 
caesarean section. Coch Lib. 2013 Jan 1

13. Hofmeyr GJ, Mathai M, Shah AN, Novikova N. Techniques for caesarean section. The Cochrane Library. 2008.

14. WHO. http://www.who.int/reproductivehealth/publications/materna 1_perinatal_health/en/Surgical techniques for caesarean section

15. Ghahiry A, Rezaei F, Karimi Khouzani R, Ashrafinia M. Comparative analysis of long-term outcomes of Misgav Ladach technique cesarean section and traditional cesarean section. Journal of Obstetrics and Gynaecology Research. 2012 Oct 1;38(10):1235-9.

16. Dumas AM, Girard R, Ayzac L, Caillat-Vallet E, Tissot-Guerraz

F, Vincent-Bouletreau A, et al. Maternal infection rates after cesarean delivery by Pfannenstiel or Joel-Cohen incision: A multicenter surveillance study. Eur J Obst \& Gyn Rep Bio. 2009;147(2):139-143. 